\title{
An Elementary Notion of Gauge Equivalence
}

\begin{abstract}
An elementary notion of gauge equivalence is introduced that does not require any Lagrangian or Hamiltonian apparatus. It is shown that in the special case of theories, such as general relativity, whose symmetries can be identified with spacetime diffeomorphisms this elementary notion has many of the same features as the usual notion. In particular, it performs well in the presence of asymptotic boundary conditions.
\end{abstract}

\section{Introduction}

The equations of motion of a classical theory are said to be underdetermined if they are not independent of one another. ${ }^{1}$ The most prominent types of theories with underdetermined equations include generally covariant theories and theories of Yang-Mills type. But there are many other examples [19].

Uniqueness of solutions fails radically in theories with underdetermined equations of motion: the family of solutions corresponding to an admissible set of initial data is infinite-dimensional-roughly speaking, such a family can be parameterized by arbitrary functions of the independent variables of the theory.

A theory is deterministic if and only if each instantaneous state is compatible with only one global history. So, in general, there is a tight connection between a failure of uniqueness of solutions in a theory and the failure of that theory to be deterministic. But this connection obtains only if we assume that distinct solutions of our theory always represent physically distinct situations. Faced with the

G. Belot

Department of Philosophy, University of Pittsburgh

Pittsburgh PA 15260, USA

E-mail: gbelot@pitt.edu

1 See [25, p. 171] for a precise characterization. 
prospect of a wholesale and dramatic failure of determinism in the presence of underdetermined equations of motion, one usually prefers to reject this assumption. Standardly one assumes instead that a theory featuring underdetermined equations involves gauge freedom - one assumes, that is, that some of the degrees of freedom of the theory are unphysical and that, except perhaps in special cases, specifying instantaneous values of all variables suffices to determine the past and future behaviour of the physical degrees of freedom.

In any classical theory, whether or not it has underdetermined equations of motion, we say that solutions are gauge equivalent if they agree as to the behaviour of all of the physical degrees of freedom. Gauge equivalence is an equivalence relation on the space of solutions of the theory. Intuitively, gauge equivalent solutions necessarily represent the same physical possibility - so the quotient space of solutions modulo gauge equivalence provides a good parameterization of the physically distinct situations described by the theory.

In a classical theory in which each generic initial data set is consistent with a unique solution, it is natural to take each solution to be gauge equivalent only to itself. But how ought one to characterize the relation of gauge equivalence in a theory with underdetermined equations of motion?

The answer to this question is pretty clear for theories given in Lagrangian form. Given a Lagrangian for a theory, one can construct a natural notion of gauge equivalence: employing by-products of the variational procedure to construct a presymplectic form on the space of solutions of the theory and taking two solutions to be gauge equivalent if and only if they can be connected by a (piecewise) null curve of this presymplectic form $[8,9,33,34] .^{2}$

For heuristic and pedagogic purposes one would like to have available an elementary notion of gauge equivalence - one which did not require recourse to a Lagrangian or to the rather technical apparatus required under the the Lagrangian approach.

Further, there exist interesting field theories that cannot be derived from a Lagrangian unless one introduces potentials or fields whose physical status is obscure. This class of theories includes the simplest possible generally covariant field theories $[28,29]$. For this reason too it would be nice to have available an elementary notion of gauge equivalence that could be applied to any field theory, whether or not it was given in Lagrangian form.

There are in fact two informal approaches to defining gauge equivalence directly in terms of the equations of a theory that one often comes across in discussions of theories with underdetermined equations of motion.

(1) Spacetime diffeomorphisms are symmetries of general relativity—so it makes sense to follow the practice of differential geometers and to consider solutions to be equivalent if and only if they are related by a diffeomorphism. Likewise, in Yang-Mills theories vertical bundle automorphisms are symmetries and so it is natural to take solutions to be gauge equivalent if and only if they are related by such a transformation. And so on.

(2) Solutions should be considered to be gauge equivalent if they induce the same initial data at some instant of time.

\footnotetext{
2 Alternatively, one can follow the Dirac constraint algorithm, which leads from a Lagrangian to a notion of gauge equivalence on the space of initial data of the Hamiltonian formulation of the theory $[10,16,19]$. This approach is closely related to the one mentioned above [24].
} 
Neither of these informal approaches is promising as a fully general characterization of gauge equivalence.

Approach (1) characterizes gauge equivalence only in special cases and hence is only a gesture towards a fully general characterization. Furthermore, it sometimes leads to unacceptable conclusions. Consider a sector of general relativity in which asymptotic boundary conditions have been imposed. Let the space of solutions be denoted by $\mathscr{S}$, the group of spacetime diffeomorphisms that preserve the asymptotic boundary conditions by $\mathscr{D}$, and the group of spacetime diffeomorphisms asymptotic to the identity at infinity by $\mathscr{D}_{0}$. In typical cases of physical interest, we find that $\mathscr{D}$ can be viewed as the product of $\mathscr{D}_{0}$ with a group $G$ that is called the asymptotic symmetry group because it can be thought of as acting geometrically at infinity. One then expects to find that $G$ acts also on $\mathscr{S} / \mathscr{D}_{0}$ and that this action is associated in the usual way with the conserved quantities that the imposition of asymptotic boundary conditions brings into existence. ${ }^{3}$ In this case, it is natural to take solutions to be gauge equivalent if and only if related by a diffeomorphism in $\mathscr{D}_{0}$ so that the space $\mathscr{S} / \mathscr{D}_{0}$ can be viewed as representing the physics of the theory without redundancy. ${ }^{4}$ But Approach (1) above would appear to recommend instead taking the space $\mathscr{S} / \mathscr{D}$ to play this role-which would efface the interesting representation of $G$ as the symmetry group of the theory.

As it stands, Approach (2) offers only a sufficient condition for gauge equivalence, not a necessary one. ${ }^{5}$ Worse, this approach implies the intuitively unacceptable result that a failure of uniqueness of solutions in a theory with underdetermined equations of motion never indicates a breakdown of physical determinism. Consider, for example, the fact that there exist maximal globally hyperbolic solutions of Einstein's field equations that admit multiple non-isometric maximal extensions. ${ }^{6}$ Such competing extensions intuitively correspond to distinct possible universes-but they count as gauge equivalent under Approach (2), since they induce the same initial data on any Cauchy surface of the original globally hyperbolic solution.

The purpose of this paper is to introduce an elementary notion of gauge equivalence that agrees with our intuitions and with the standard Lagrangian notion in cases of central interest. Aside from a couple of technicalities, the notion introduced is as follows: one wants to consider two solutions to be gauge equivalent if they (a) induce the same initial data at an instant of time and (b) are related by a symmetry of the theory; one takes gauge equivalence to be the equivalence relation generated by this requirement.

In Section 2 below the relevant framework and definitions are presented. In Section 3 the approach is applied to the class of theories, such as vacuum general relativity, in which spacetime diffeomorphisms are the only source of gauge freedom. Finally, Section 4 includes several remarks regarding the prospects for

\footnotetext{
${ }^{3}$ For examples see $[3,20]$.

4 The Lagrangian approach to gauge equivalence leads to this same conclusion [3,20].

${ }^{5}$ For solution $\Phi_{1}$ can induce the same initial data as solution $\Phi_{2}$ at one instant while solution $\Phi_{2}$ induces the same initial data as solution $\Phi_{3}$ at another instant without there being any instant at which $\Phi_{1}$ and $\Phi_{3}$ induce the same initial data. But since gauge equivalence is presumably an equivalence relation, we will nonetheless want to count $\Phi_{1}$ and $\Phi_{3}$ as gauge equivalent.

6 Taub and Misner spacetimes are examples of globally hyperbolic spacetimes admitting inequivalent extensions [17, §5.8]. For further discussion, examples, and references see [7].
} 
extending the present approach and concerning the relation between this approach and the standard Lagrangian approach.

\section{Gauge Equivalence}

For present purposes, we can take a field theory to consist of the following elements. $^{7}$

(a) A connected $n$-dimensional manifold (without boundary), $V$, the spacetime of the theory.

(b) A set $\Theta$ of solution-independent tensors on $V$. We call these the fixed fields of the theory. ( $\Theta$ is often the empty set in cases of interest.)

(c) A set $\left\{\phi_{1}, \ldots, \phi_{k}\right\}$ of dynamical fields on spacetime, each of which is specified by specifying a type of tensor on $V$. A particular configuration of these fields is denoted $\Phi=\left(\phi_{1}, \ldots, \phi_{k}\right)$.

(d) An infinite-dimensional manifold $\mathscr{K}$ consisting of the kinematically possible $\Phi . \mathscr{K}$ is determined by specifying the required degree of differentiability, asymptotic behaviour, etc. of the fields of the theory.

(e) A set of differential equations, $\Delta(\Phi ; \Theta)$ that determines a subspace $\mathscr{S} \subset \mathscr{K}$, the space of solutions of the theory. The fixed fields play the role of parameters rather than variables in the equations $\Delta$. Any derivative operators occurring in $\Delta$ must be definable in terms of the elements listed above.

Typically, $\mathscr{S}$ will contain mild singularities and so will not be itself be a manifold $[1,2,21]$. But for each solution $\Phi \in \mathscr{S}$, the set of solutions of the same symmetry type as $\Phi$ will form a manifold, and $\mathscr{S}$ can be viewed as the disjoint union of the manifolds that arise in this way, which are called the strata of $\mathscr{S}$. However, spaces of this type share many features with manifolds [26] —e.g., such spaces support a notion of smooth function, and it makes sense to speak of the tangent space at a point in such a space.

As usual, the symmetry group, $\mathscr{G}$, of a field theory is the group of diffeomorphisms from $\mathscr{K}$ to itself that map solutions to solutions and that are suitably local on $V .{ }^{8}$ The relevant notion of locality is made precise in Remark 1 below. We call the elements of $\mathscr{G}$ the symmetries of the theory and write $\gamma \cdot \Phi$ for the result of acting on $\Phi \in \mathscr{S}$ by a symmetry $\gamma \in \mathscr{G}$.

The notion of gauge equivalence developed here requires one further essential notion, that of an initial data surface. This notion will function informally in the present paper. Let us say that a hypersurface $\Sigma \subset V$ (i.e., an $(n-1)$-dimensional submanifold of $V$ ) cuts across $V$ if $V / \Sigma$ consists of two connected components. Intuitively, a hypersurface $\Sigma$ that cuts across $V$ counts as an initial data surface relative to a solution $\Phi$ if: (i) the initial data that $\Phi$ induces on $\Sigma$ come as close to determining $\Phi$ as is possible given the nature of the equations of the theory; and (ii) $\Sigma$ is asymptotically well-behaved. For purposes of illustration, let us assume that our theory involves as one of its fields a Lorentzian metric $g$ and that all of

\footnotetext{
7 No attempt is made to be maximally general here. See Section 4 below for further discussion.

${ }^{8}$ Here and below we restrict attention to diffeomorphisms in the connected component of the identity.
} 
the other fields are well-behaved relative to $g$. If $g$ is a fixed field and is globally hyperbolic, then it is natural to identify the initial data surfaces of the theory with the Cauchy surfaces of $g$; if $(V, g)$ is spatially open, then we will also require initial data surfaces to be well-behaved at spatial infinity. Similarly, if $g$ is globally hyperbolic but is a dynamic field it will again be natural to take as our initial data surfaces the (asymptotically well-behaved) Cauchy surfaces of $(V, g) \cdot{ }^{9}$ There also exist cases in which it is natural to consider certain partial Cauchy surfaces of $g$ as initial data surfaces-e.g., in the asymptotically anti-de Sitter sector of general relativity no Cauchy surfaces exist but one has strong existence and uniqueness results for initial data posed on certain spacelike surfaces with good asymptotic behaviour [12]

In the following we will always assume that our theory has been supplemented by a suitable notion of initial data surface.

Let us fix for consideration a field theory with space of solutions $\mathscr{S}$. Intuitively, $\Phi_{1}, \Phi_{2} \in \mathscr{S}$ are gauge equivalent if and only if the formalism of the theory more or less forces us to regard them as corresponding to a single physical situation. Let us begin to make this idea more precise.

Definition 1 Let $\Phi \in \mathscr{S}$ be a solution. A symmetry $\gamma \in \mathscr{G}$ of the theory is a spoiler relative to $\Phi$ if there exists a hypersurface $\Sigma \subset V$ such that: (i) $\Sigma$ is an initial data surface relative to $\Phi$; and (ii) there exists an open neighbourhood $U$ of $\Sigma$ in $V$ on which $\gamma$ acts as the identity (in the sense that for any solution $\Phi,\left.\Phi\right|_{U}=\left.\gamma \cdot \Phi\right|_{U}$ ). We say that $\Phi_{1}, \Phi_{2} \in \mathscr{S}$ are spoiler-related if there exists a spoiler $\gamma$ relative to $\Phi_{1}$ such that $\Phi_{2}=\gamma \cdot \Phi_{1}$. In this case we write $\Phi_{1} * \Phi_{2}$.

Familiar examples of spoilers arise as follows. In general relativity, the symmetries of the theory are diffeomorphisms and scale transformations [32]. If $\Sigma$ is an initial data surface relative to $g \in \mathscr{S}$, then any diffeomorphism that acts as the identity on an open neighbourhood of $\Sigma$ is a spoiler relative to $g$ (and all spoilers are of this form). In Maxwell theory set in Minkowski spacetime, the spoilers are just the gauge transformation of the form $A \mapsto A+\Lambda$ with $\Lambda$ vanishing on an open neighbourhood of an initial data surface.

If $\Phi_{1}, \Phi_{2} \in \mathscr{S}$ are related by a spoiler $\gamma$, then these solutions induce the same initial data on any hypersurface $\Sigma$ in virtue of which $\gamma$ is a spoiler. Aversion to indeterminism provides reason to view $\Phi_{1}$ and $\Phi_{2}$ as corresponding to the same physical situation. And there is no obstacle to doing so, since these solutions are related by a symmetry of the theory and thus are suited to represent exactly the same situations.

Of course, 'corresponding to the same situation' ought to be an equivalence relation on the space of solutions. So while two solutions correspond to the same situation if they are related by a spoiler, the converse is not true: in the case where $\Phi_{1} * \Phi_{2}$ and $\Phi_{2} * \Phi_{3}$, we will want to regard $\Phi_{1}$ and $\Phi_{3}$ as necessarily corresponding to the same physical situation, whether or not they are related by a spoiler. So, we certainly want to say that two solutions $\Phi$ and $\Phi^{\prime}$ are gauge equivalent if we can find solutions $\Phi_{1}, \ldots, \Phi_{k}$ such that $\Phi=\Phi_{1}, \Phi^{\prime}=\Phi_{k}$, and $\Phi_{i} * \Phi_{i+1}$ for each $i=1, \ldots, k-1$.

\footnotetext{
${ }^{9}$ Of course when $g$ is fixed, the question whether $\Sigma \subset V$ is an initial data surface is a solutionindependent one, but this is no longer the case when $g$ is a dynamic field.
} 
It is helpful to build a further requirement into our official notion of gauge equivalence. ${ }^{10}$ We motivate this as follows. Specifying an equivalence relation on a set $X$ is equivalent to specifying a partition of $X$ by subsets. When $X$ carries additional structure it is natural to restrict attention to equivalence relations corresponding to partitions that respect the structure of $X$. Since $\mathscr{S}$ is a sort of smooth object, it makes sense to restrict attention to equivalence relations that are smooth in the sense that their equivalence classes form submanifolds of (the strata of) $\mathscr{S}$.

Definition 2 Gauge equivalence is the weakest smooth equivalence relation on $\mathscr{S}$ that is stronger than $*$, when such an equivalence relation exists (otherwise the notion is undefined).

In other words, when defined, gauge equivalence is the weakest equivalence relation $R$ on $\mathscr{S}$ such that: (i) the equivalence classes of $R$ form submanifolds of $\mathscr{S}$; and (ii) $\Phi * \Phi^{\prime}$ implies $R\left(\Phi, \Phi^{\prime}\right)$.

In order to appraise the interest of this definition, it is necessary to see what results it underwrites in cases of interest. An important class of examples will be considered in the next section.

Remark 1 (Locality) Let $\mathscr{G}$ be a group of diffeomorphisms from $\mathscr{K}$ to itself such that it makes sense to speak of the infinitesimal generators of the one-parameter subgroups of $\mathscr{G}$. Such a generator $\xi$ will be a vector field on $\mathscr{K}$. In the present setting, a vector $\delta \Phi \in T_{\Phi} \mathscr{K}, \Phi \in \mathscr{K}$, can be identified locally with a function on $V$. $G$ is local in the sense required for the definition of a symmetry of a field theory if for every infinitesimal generator $\xi$ of a one-parameter subgroup of $\mathscr{G}$, the value of $\xi(\Phi) \in T_{\Phi} \mathscr{K}$ when evaluated at a point $x \in V$ depends only on the values at $x$ of $\Phi$ and finitely many of its derivatives.

\section{Application: Spatiotemporal Symmetries and Gauge Equivalence}

Consider a field theory set in spacetime $V$ and with space of solutions $\mathscr{S}$.

Let $\operatorname{Diff}(V)$ be the group of diffeomorphisms from $V$ to itself. ${ }^{11}$ Let $d: \Phi \mapsto$ $d \cdot \Phi$ denote the obvious action of $\operatorname{Diff}(V)$ on $\mathscr{K}$ (according to which a $d$ acts on each component field of $\Phi$ in the usual way). We say that $d \in \operatorname{Diff}(V)$ is a spatiotemporal symmetry of our theory it maps solutions to solutions (spacetime diffeomorphisms act locally, so a spacetime symmetry is a symmetry in the sense discussed above).

We call a theory generally covariant if every $d \in \operatorname{Diff}(V)$ is a spatiotemporal symmetry. We call a theory locally generally covariant if each compactly supported element of $\operatorname{Diff}(V)$ is a spatiotemporal symmetry. Within the present framework, a nontrivial theory is locally generally covariant if and only if it features no fixed fields. Typical examples of theories that are locally generally covariant but not generally covariant include theories that have no fixed fields and that feature

\footnotetext{
${ }^{10}$ Whether it is in fact necessary to impose this as a separate requirement will be discussed below in Section 3.2.

11 Again, we restrict attention here and throughout to diffeomorphisms in the connected component of the identity.
} 
(asymptotic) boundary conditions-in such theories the group of spacetime symmetries is the subgroup $\mathscr{D}$ of $\operatorname{Diff}(V)$ consisting of diffeomorphisms that preserve the (asymptotic) boundary conditions.

In the present Section our aim is to investigate gauge equivalence that arises from (local) general covariance. So we restrict attention for the remainder of this Section to theories all of whose spoilers arise as spatiotemporal symmetries. This includes, e.g., any theory with a well-posed initial value problem (when the initial value problem is well-posed the only spoiler is the trivial one-which can be identified with the transformation of $\mathscr{K}$ induced by the identity diffeomorphism on $V$ ) and vacuum general relativity (as noted above, the symmetry group of Einstein's field equations is generated by spacetime diffeomorphisms and scale transformations-but no nontrivial scale transformation is a spoiler).

With this restriction in place, we find the following picture.

(1) In a field theory featuring a Lorentzian metric $g$ as a fixed field each solution is gauge equivalent only to itself.

(2) In a well-behaved field theory, gauge equivalent solutions are related by a spatiotemporal symmetry of the theory.

(3) In a well-behaved generally covariant field theory in which the initial data surfaces are compact, one expects to find that solutions are gauge equivalent if and only if related by a diffeomorphism.

(4) In a well-behaved locally generally covariant theory featuring asymptotic boundary conditions, one expects to find that two solutions are gauge equivalent if and only if related by a diffeomorphism that acts as the identity at infinity.

These claims are made precise, established, and illustrated in the next subsection. Section 3.2 takes up the question whether it is really necessary to build into the definition of gauge equivalence the condition that it be a smooth equivalence relation.

\subsection{Proofs and Illustrations}

Let us fix a field theory with spacetime $V$, and space of solutions $\mathscr{S}$ and assume that the only spoilers arise as spatiotemporal symmetries.

Proposition 1 If the theory features a Lorentzian metric as a fixed field, then every solution is gauge equivalent only to itself.

Proof Consider the equivalence relation $I$ on $\mathscr{S}$ according to which every solution is related only to itself. $I$ is smooth (its equivalence classes are zero-dimensional submanifolds of $\mathscr{S}$ ). And $\Phi * \Phi^{\prime}$ implies $I\left(\Phi, \Phi^{\prime}\right)$ (in the present case all spoilers are spatiotemporal symmetries, and all spatiotemporal symmetries are isometries of $g$-but no non-trivial isometry of a Lorentzian metric acts as the identity on an open set). Further, $I$ is the weakest equivalence relation with these features ( $I$ is the weakest equivalence relation on $\mathscr{S}$ ).

Note that the same conclusion would not follow if we assumed simply that the theory involved a nontrivial fixed field: a symplectic form, for instance, admits nontrivial symmetries with compact support. 
Definition 3 Let $D$ be a subgroup of $\operatorname{Diff}(V)$. We say that $D$ acts nicely on $\mathscr{S}$ if for each $\Phi \in \mathscr{S}$ the orbit $D \cdot \Phi:=\{d \cdot \Phi: d \in D\}$ is a submanifold of $\mathscr{S}$.

One expects of course that in a well-behaved theory the group $\mathscr{D}$ of spatiotemporal symmetries acts nicely on the space of solutions.

Proposition 2 Suppose that $\mathscr{D}$ acts nicely on $\mathscr{S}$. Then if two solutions are gauge equivalent then they are are related by a spatiotemporal symmetry.

Proof Consider the equivalence relation $R$ according to which two solutions are equivalent if and only if related by a spatiotemporal symmetry. By assumption $R$ is smooth. And $\Phi * \Phi^{\prime}$ implies $R\left(\Phi, \Phi^{\prime}\right)$ (since in the present context every spoiler is a spatiotemporal symmetry). So $R$ is stronger than gauge equivalence, should the latter notion be well-defined. It follows that if two solutions are gauge equivalent then they are related by a spatiotemporal symmetry.

Definition 4 Let $M$ be a manifold, possibly with boundary and let $S_{1}$ and $S_{2}$ be subsets of $M$. We say that $S_{1}$ and $S_{2}$ are well-separated if there exist compact $K_{1}, K_{2} \subset M$ such that $S_{1}$ is contained in $K_{1}^{o}$ (the interior of $K_{1}$ ), $K_{1}$ is contained in $K_{2}^{o}$, and $S_{2}$ is disjoint from $K_{2}$.

For example, in a spatially compact globally hyperbolic spacetime, nonintersecting Cauchy surfaces are well-separated.

Proposition 3 In a generally covariant field theory theory in which Diff( $(V)$ acts nicely, the initial data surfaces are compact, and every solution admits wellseparated initial data surfaces, two solutions are gauge equivalent if and only if related by an element of Diff $(V)$.

Proof Consider the equivalence relation $R$, according to which two solutions are equivalent if related by a spacetime diffeomorphism. Let us denote the equivalence classes of $R$ by $[\Phi]_{R}$. By assumption, $\operatorname{Diff}(V)$ acts nicely, so $R$ is smooth. And by assumption, every spoiler is a spatiotemporal symmetry, so $\Phi * \Phi^{\prime}$ implies $R\left(\Phi, \Phi^{\prime}\right)$. In order to show that $R$ is the relation of gauge equivalence, we must show that $R$ is the weakest equivalence relation on $\mathscr{S}$ with these two features.

Consider a smooth equivalence relation $S$ on $\mathscr{S}$ that is stronger than $*$. Let us denote the equivalence classes of $S$ by $[\Phi]_{S}$.

Fix a solution $\Phi \in \mathscr{S}$. By assumption $[\Phi]_{R}$ and $[\Phi]_{S}$ are submanifolds of $\mathscr{S}$ and so determine well-defined tangent subspaces $T_{\Phi}[\Phi]_{R}, T_{\Phi}[\Phi]_{S} \subset T_{\Phi} \mathscr{S}$. In order to establish the Proposition, it suffices to show that $T_{\Phi}[\Phi]_{R} \subseteq T_{\Phi}[\Phi]_{S}$ - for from this it follows directly that $[\Phi]_{R} \subseteq[\Phi]_{S}$.

In order to show that $T_{\Phi}[\Phi]_{R} \subseteq T_{\Phi}[\Phi]_{S}$ we choose an arbitrary $\delta \Phi \in T_{\Phi}[\Phi]_{R}$ and show that $\delta \Phi \in T_{\Phi}[\Phi]_{S}$ by showing that it can be written as the sum of two elements of $T_{\Phi}[\Phi]_{S}$.

By the definition of $R$, there is a flow $\xi$ on $V$ and a complete vector field $v_{\xi}$ on $V$ that generates $\xi$ with $\delta \Phi=\mathscr{L}_{v_{\xi}} \Phi$ (the Lie derivative of $\Phi$ along $v_{\xi}$ ).

Choose $\Sigma_{1}, \Sigma_{2} \subset V$ that are initial data surfaces relative to $\Phi$ and which are well-separated by compact sets $K_{1}, K_{2} \subset V$. We can find a smooth $f: V \rightarrow \mathbb{R}$ so that $f(x)=1$ for $x \in K_{1}$ and $f(x)=0$ for $x \in V / K_{2}^{o}$ (since these are disjoint closed sets). Define new vector fields $v_{1}$ and $v_{2}$ on $V$ by $v_{1}:=(1-f) v_{\xi}$ and $v_{2}=f v_{\xi}$. 
Note the following facts: (a) each $v_{i}$ vanishes on an open neighbourhood of $\Sigma_{i}$ $(i=1,2)$; (b) $v_{\xi}=v_{1}+v_{2}$.

We claim that $v_{1}$ and $v_{2}$ are complete vector fields. Indeed, it is a basic result that if $v$ is a vector field on a manifold $M$ and $K$ a compact subset of $M$ and the integral curve of $v$ through a point $x \in K$ is defined only for $t$ smaller than some $\tau \in \mathbb{R}$, then there must be a time $t_{1}<\tau$ after which the curve leaves $K$ and does not return [23, Theorem IV.2.3]. A straightforward corollary is that if $v$ is a complete vector field on $M$ and $v^{\prime}$ is a vector field on $M$ that agrees with $v$ outside of some compact set $K \subset M$ then $v^{\prime}$ is also complete. In our case, $v_{1}$ differs from the complete vector field $v_{\xi}$ only on the compact set $K_{2}$ while $v_{2}$ differs from the complete vanishing vector field only on $K_{2}$.

So $v_{1}$ and $v_{2}$ generate flows $\xi_{1}$ and $\xi_{2}$ on $V$-and that by fact (a) above, these flows consist of spoilers. It then follows that the tangent vectors $\delta_{1} \Phi, \delta_{2} \Phi \in \mathscr{S}$ defined by $\delta_{i} \Phi:=\mathscr{L}_{v_{i}} \Phi, i=1,2$, lie in $T_{\Phi}[\Phi]_{S}$ (since $S$ is stronger than $*$ ). And since the correspondence $v \mapsto \mathscr{L}_{v} \Phi$ is linear in $v$, we see that fact (b) implies that $\mathscr{L}_{v_{\xi}} \Phi=\mathscr{L}_{v_{0}} \Phi+\mathscr{L}_{v_{1}} \Phi$. So we have decomposed $\delta \Phi$ as a sum of two elements of $T_{\Phi}[\Phi]_{S}$.

Example 1 (Spatially Compact Vacuum General Relativity.) Consider the sector of general relativity in which $V$ has the form $\mathbb{R} \times M$ ( $M$ compact), kinematically possible $g$ are required to be globally hyperbolic, and the equations of motion are the vacuum Einstein field equations. Modulo certain reasonable technical assumptions, one finds that $\operatorname{Diff}(V)$ acts nicely on $\mathscr{S}[21, \S 5]$. It is natural to identify initial data surfaces with Cauchy surfaces (which are, of course, themselves compact). And any two disjoint Cauchy surfaces are well-separated. So Proposition 3 applies, and we find that solutions are gauge equivalent if and only if related by a spacetime diffeomorphism. The corresponding result holds in the Lagrangian approach to gauge equivalence [33, p. 145].

Let us next consider locally generally covariant theories in which full general covariance is broken by the presence of asymptotic boundary conditions. Typical examples can be cast into the following form. One adjoins to the physical spacetime manifold $V$ a non-physical $(n-1)$-dimensional boundary $\partial V$; kinematically possible $\Phi$ are required to exhibit some sort of good behaviour as they approach $\partial V$. We say that a theory featuring asymptotic boundary conditions has been put in standard form if it has been formulated in these terms.

We write $\bar{V}$ for $V \cup \partial V$. More generally, for any $U \subset V$ we write $\bar{U}$ for the closure of $U$ in $\bar{V}$ and write $\partial U$ for $\bar{U} / U$. Typically $\partial V$ can be viewed as equipped with some sort of solution-independent geometry that picks out a distinguished class of $(n-2)$-dimensional subsets of $\partial V$. We say will say that a hypersurface $\Sigma \subset V$ has good asymptotic behaviour if $\partial \Sigma$ is one of these distinguished subsets.

We denote by $\mathscr{D}$ the group of spatiotemporal symmetries of our theory and by $\mathscr{D}_{0}$ the group of spatiotemporal symmetries asymptotic to the identity at $\partial V$. In typical examples $\mathscr{D}_{0}$ is a proper subgroup of $\mathscr{D}$ which is a proper subgroup of $\operatorname{Diff}(V)$. In the examples that arise in practice one typically also finds that $\mathscr{D}_{0}$ is a normal subgroup of $\mathscr{D}$ and that the quotient group $G$ can be thought of as acting geometrically at $\partial V$ - when it exists, $G$ is called the asymptotic symmetry group.

Proposition 4 Consider a locally generally covariant theory featuring asymptotic boundary conditions that has been cast into standard form. Suppose that every 
spoiler acts as the identity at $\partial V$, that $\mathscr{D}_{0}$ acts nicely on $\mathscr{S}$, and that every solution admits initial data surfaces $\Sigma_{1}, \Sigma_{2} \subset V$ with good asymptotic behaviour such that $\bar{\Sigma}_{1}$ and $\bar{\Sigma}_{2}$ are well-separated in $\bar{V}$. Then solutions are gauge equivalent if and only if related by an element of $\mathscr{D}_{0}$.

Proof A straightforward adaptation of the proof of the preceding Proposition.

Example 2 (General Relativity with Asymptotic Flatness Imposed at Spatial Infinity.) Consider the sector of vacuum general relativity in which asymptotic flatness is imposed at spatial infinity in the sense of [1]. Here $V$ is $\mathbb{R}^{4}, \partial V$ is the unit timelike hyperboloid $\mathscr{H}$ in Minkowski spacetime, and the asymptotic symmetry group is the Poincare group. Modulo certain reasonable assumptions one finds that $\mathscr{D}_{0}$ acts nicely on $\mathscr{S}$. In this setting it is natural to count a cauchy surface $\Sigma \subset V$ as an initial data surface if and only if $\partial \Sigma \subset \partial V$ is isomorphic to the intersection of $\mathscr{H}$ with a spacelike hyperplane in Minkowski spacetime. Any solution can be foliated by a family $\left\{\Sigma_{t}\right\}$ of initial data surfaces such that the $\partial \Sigma_{t}$ foliate $\partial V$. So it is certainly possible to find initial data surfaces $\Sigma_{1}$ and $\Sigma_{2}$ such that $\bar{\Sigma}_{1}$ and $\bar{\Sigma}_{2}$ are well-separated in $\bar{V}$. Furthermore each spoiler is in $\mathscr{D}_{0}$ (each spatiotemporal symmetry induces an action of the Poincaré group on the set of frames at infinity; only the identity transformation acts pointwise on a set of the form $\partial \Sigma$ for $\Sigma$ an initial data surface). So the hypotheses of Proposition 4 hold, and we conclude that solutions are gauge equivalent if and only if related by an element of $\mathscr{D}_{0}$. We find that the space of solutions modulo the relation of gauge equivalence carries a representation of the Poincare group-which action is associated in the usual way with the conserved quantities that the imposition of asymptotic flatness at spatial infinity brings in to existence. The same results hold within the Lagrangian approach [3].

Within general relativity, the setting of asymptotically simple spacetimes provides further examples of asymptotic boundary conditions. Recall that a fourdimensional vacuum solution $(V, g)$ of the Einstein field equations with cosmological constant is asymptotically simple if there is a non-empty spacetime $(\bar{V}, \bar{g})$ with boundary $\partial \bar{V}$ such that:

1. $V$ is the interior of $\bar{V}$.

2. Each null geodesic of $(V, g)$ has a past endpoint and a future endpoint on $\partial \bar{V}$.

3. There exists a smooth $\Omega: \bar{V} \rightarrow \mathbb{R}$ such that:
(a) $\bar{g}=\Omega^{2} g$ on $V$.
(b) $\Omega>0$ on $V$.
(c) $\Omega=0$ and $d \Omega \neq 0$ on $\partial \bar{V}$.

In this case $(\bar{V}, \bar{g})$ is called a conformal completion of $(V, g)$ and $\partial \bar{V}$ is called conformal infinity. 12

The topology of $\partial \bar{V}$ is determined by $(V, g)$ but its geometry is determined only up to a conformal factor. Thus, in any conformal completion of anti-de Sitter spacetime $\partial \bar{V}$ has the topology of $\mathbb{R} \times S^{2}$ and carries a Lorentzian metric conformally equivalent to that of the three-dimensional Einstein static universe. In any conformal completion of Minkowski spacetime, $\partial \bar{V}$ falls into two pieces, each with the topology of $\mathbb{R} \times S^{2}$ and each carrying a pair $(\gamma, n)$ where $\gamma$ is a metric

\footnotetext{
12 For recent results and references see [13,14].
} 
of signature $(0,-,-)$ and $n$ is a null vector field of $\gamma$ (under change of conformal factor we have $\left.\Omega:(g, n) \mapsto\left(\Omega^{2} g, \Omega^{-1} n\right)\right)$. In any conformal completion of de Sitter spacetime, $\partial \bar{V}$ falls into two pieces, each with the topology of $S^{3}$ and equipped with a Riemannian metric conformally equivalent to the standard constant curvature metric on $S^{3}$.

Let $\left(V, g_{0}\right)$ be one of these three spacetimes of constant curvature. Then we construct a sector of general relativity in which solutions are required to be asymptotic to $\left(V, g_{0}\right)$ at conformal infinity as follows. The spacetime of our theory is chosen to be $V$ and the metric field $g$, the only field of the theory, is governed by the vacuum Einstein field equation with cosmological constant of the sign corresponding to $\left(V, g_{0}\right)$. Fix a conformal completion $\left(\bar{V}, \bar{g}_{0}\right)$ of $\left(V, g_{0}\right)$ and count a metric $g$ on $V$ as kinematically possible if and only if it has a conformal completion of the form $(\bar{V}, \bar{g})$ such that $\bar{g}_{0}$ and $\bar{g}$ induce conformally related geometries on $\partial \bar{V}$. We call the three resulting sectors of general relativity the asymptotically anti-de Sitter sector, the asymptotically Minkowski sector, and the asymptotically de Sitter sector.

In each case we find that the group $\mathscr{D}$ of spatiotemporal symmetries is the semi-direct product of the group $\mathscr{D}_{0}$ consisting of symmetries that act as the identity at $\partial \bar{V}$ with an asymptotic symmetry group $G$-each $d \in \mathscr{D}$ induces a $\bar{d} \in G$ that acts as a conformal symmetry on $\partial \bar{V}$. In the anti-de Sitter/Minkowski/de Sitter case case, $G$ is the the anti-de Sitter/Bondi-Metzner-Sachs/de Sitter group. ${ }^{13}$

By analogy with the case in which asymptotic flatness is imposed at spatial infinity, one would expect that in each of our three cases solutions should be gauge equivalent if and only if related by an element of $\mathscr{D}_{0}$ and that the space of solutions modulo gauge equivalence should carry a representation of the asymptotic symmetry group.

Example 3 (Asymptotically anti-de Sitter spacetimes.) Here $\partial \bar{V}$ is conformally equivalent to the three-dimensional Einstein static universe. Although solutions in this sector are not globally hyperbolic, there is an appropriate notion of initial data surface $[12, \S 5.1]$ : there exists a class of spacelike surfaces with good asymptotic behaviour such that strong existence and uniqueness results hold for initial data posed on such surfaces. Such initial data surfaces intersect $\partial \bar{V}$ in spacelike twospheres and it is possible to foliate a solution by such surfaces so that $\partial \bar{V}$ is foliated by their limits at spatial infinity—so it is easy to find initial data surfaces whose closures in $\bar{V}$ are well-separated. Furthermore, the group of conformal symmetries of $\partial \bar{V}$ is the anti-de Sitter group, so it is clear that any spoiler must act as the identity on $\partial \bar{V}$ (since no element of the de Sitter group fixes a sphere point-wise). So if we assume that $\mathscr{D}_{0}$ acts nicely on $\mathscr{S}$, then Proposition 4 tells us that solutions are gauge equivalent if and only if related by a diffeomorphism in $\mathscr{D}_{0}$. It then follows that we have a representation of the anti-de Sitter group acting on the space of solutions modulo gauge equivalence. One can reach the same conclusion via the Lagrangian approach [20] - and one then finds that the action of the asymptotic symmetry group on the space of solutions modulo gauge equivalence generates conserved quantities in the usual way.

Example 4 (Asymptotically Minkowski spacetimes.)

\footnotetext{
${ }^{13}$ See, e.g., [18] or [4] for the anti-de Sitter case, [15] for the Minkowski case, and [6] for the de Sitter case.
} 
This case, which differs from the case of Example 2 above because asymptotic flatness is now imposed at null infinity rather than at spatial infinity, is a bit more subtle. Solutions in this sector are globally hyperbolic, so one might suppose that initial data surfaces should be taken to coincide with Cauchy surfaces. But Cauchy surfaces do not have good asymptotic behaviour: they do not intersect $\partial \bar{V} .{ }^{14}$ It follows that no Cauchy surface is contained in a compact set in $\bar{V}$ and so it is impossible to apply the techniques of proof employed above.

There is a way around this difficulty. Once one stipulates that a solution is asymptotically Minkowski, one expects that fixing initial data on any spacelike hypersurface that intersects either component of $\partial \bar{V}$ transversally should fix the global state of the solution. ${ }^{15}$ So let us take as our initial data surfaces partial Cauchy surfaces with such asymptotic behaviour. It is then easy to find initial data surfaces whose closures are well-separated in $\bar{V}$. And one also finds that any spoiler acts as the identity at $\partial \bar{V} .{ }^{16}$ So if we assume that $\mathscr{D}_{0}$ acts nicely on $\mathscr{S}$, then Proposition 4 suffices to establish that asymptotically Minkowski solutions are gauge equivalent if and only if related by an element of $\mathscr{D}_{0}$. One then again finds that the space of solutions modulo gauge equivalence carries a representation of the asymptotic symmetry group - in this case, the BMS group. The same conclusion can be arrived at via a Lagrangian analysis, where one finds the usual connection between asymptotic symmetries and conserved quantities $[3, \S 5]$.

Example 5 (Asymptotically de Sitter spacetimes.) This case is again not entirely straightforward. Every asymptotically de Sitter solution is spatially compact and globally hyperbolic. Again, the most straightforward approach is to identify initial data surfaces with Cauchy surfaces. If we adopt this approach and further assume that $\mathscr{D}$ acts nicely on the space of solutions, then an argument directly analogous to the proof of Proposition 3 above suffices to show that asymptotically de Sitter solutions are gauge equivalent if and only if related by a spacetime diffeomorphism $d$ that preserves the asymptotic boundary conditions-with no requirement that $d$ be asymptotic to the identity at conformal infinity. It follows that we do not have a representation of the group of asymptotic symmetries on the space of solutions modulo gauge transformations. In the Lagrangian case, too, one can reach this conclusion (by again adapting the argument from the spatially compact globally hyperbolic case) if one employs Cauchy surfaces in constructing the presymplectic form on the space of solutions. ${ }^{17}$

This is unfortunate as parity with the preceding cases suggests that here too we should obtain a representation of the group of asymptotic symmetries on the space of solutions modulo gauge equivalence [30, §4].

There is a way to achieve this result. Comparison with the preceding examples suggests that we should look for a class of initial data surfaces with good asymptotic behaviour at $\partial V$ (in asymptotically de Sitter spacetimes Cauchy surfaces do not reach $\partial V$ ). To this end, let us take as our initial data surfaces those partial

\footnotetext{
14 Note that we have not included a point $i^{0}$ representing spatial infinity in our conformal completion of spacetime. In general, asymptotically Minkowski spacetimes are not well-behaved at $i^{0}$; see, e.g., $[13, \S 5]$.

15 This intuition outstrips extant results. For discussion see [11, §3.3].

16 This follows from, e.g., the discussion of [15, pp. 33-34].

17 See Section 4 below for the role that a choice of hypersurface plays in the Lagrangian approach.
} 
Cauchy surfaces that cut across $V$ and that intersect one of the other component of $\partial \bar{V}$ nicely in two-spheres. These will be analogous to the Euclidean partial cauchy surfaces in de Sitter spacetime (see $[17, \S 5.2]$ ); one again hopes that strong existence and uniqueness results can be proved for initial data posed on such surfaces within the context of asymptotically de Sitter boundary conditions. If we assume that $\mathscr{D}_{0}$ acts nicely on $\mathscr{S}$, then Proposition 4 will again suffice to show that solutions are gauge equivalent if and only if related by a diffeomorphism in $\mathscr{D}_{0}$. It is natural to suspect that one could reach the same conclusion on the Lagrangian side if one constructed the presymplectic form via integration over such non-Cauchy initial data surfaces. ${ }^{18}$

Example 6 (Asymptotic flatness at spatial infinity, again.) There exist a number of ways of imposing asymptotic flatness at spatial infinity. In the approach of [5], as in the approach discussed above in Example 2, spatial infinity has the structure of the unit hyperboloid in Minkowski spacetime. And one again finds that $\mathscr{D}$ is the semi-direct product of $\mathscr{D}_{0}$ with an asymptotic symmetry group $G$. But in this setting, rather than being the Poincaré group, $G$ is the semi-direct product of a group of supertranslations with the Lorentz group. Since in this case the group of supertranslations is parameterized by arbitrary smooth functions on the unit hyperboloid, the condition that all spoilers lie in $\mathscr{D}_{0}$ fails. One expects, in fact, that solutions will count as gauge equivalent if related by an element of $\mathscr{D}_{0}$ or a supertranslation-so that the space of solutions modulo gauge equivalence will carry a representation of the Lorentz group.

\subsection{Simplifying the Definition of Gauge Equivalence}

According to Definition 2, gauge equivalence is the weakest equivalence relation on $\mathscr{S}$ (the space of solutions of a theory) that: (i) extends the relation $*$ (the relation of being related by a spoiler); and (ii) has equivalence classes that form submanifolds of $\mathscr{S}$.

It is natural to wonder whether requirement (ii) plays an essential role. Let $S$, be the transitive closure of $*$-i.e., $S$ is the weakest equivalence relation on $\mathscr{S}$ that extends $* .{ }^{19}$ Writing $[\Phi]_{S}$ for the $S$-equivalence class of $\Phi \in \mathscr{S}$ and $[\Phi]$ for the set of solutions gauge equivalent to $\Phi$, we can pose our question this way: Do we find that for theories of central interest, $[\Phi]_{S}=[\Phi]$ for all solutions $\Phi$ ?

The answer is 'yes' - at least, if we are satisfied with a heuristic argument that involves assuming that a result true of finite-dimensional manifolds holds for $\mathscr{S}$.

So we can consider flows (i.e., one-parameter groups of diffeomorphisms) on $\mathscr{S}$ consisting of spoilers. Let us say that solutions $\Phi_{1}$ and $\Phi_{2}$ are flow-spoiler related if they are related by a spoiler that is a member of a flow of spoilers. And let us consider the equivalence relation $\mathscr{F}$ on $\mathscr{S}$ that is the transitive closure of being flow-spoiler related, denoting the equivalence classes of this relation by $[\Phi]_{\mathscr{F}}$.

For theories of interest, if $\mathscr{F}$ is smooth, then $[\Phi]_{S}=[\Phi]$. For in a field theory satisfying the hypotheses of Propositions 3 or 4 , it follows immediately from the

\footnotetext{
18 This question is to be treated in forthcoming work by Stefan Hollands and Sebastian Jaeger.

19 So solutions $\Phi, \Phi^{\prime} \in \mathscr{S}$ are $S$-related if and only if there exist solutions $\Phi_{1}, \ldots, \Phi_{n}$ such that $\Phi=\Phi_{1}, \Phi^{\prime}=\Phi_{n}$ and for each $1 \leq i \leq n-1, \Phi_{i} * \Phi_{i+1}$.
} 
definitions of the equivalence relations involved that $[\Phi]_{\mathscr{F}} \subseteq[\Phi]_{S} \subseteq[\Phi]$, for any solution $\Phi$. Further, letting $D$ denote either $\operatorname{Diff}(V)$ or $\mathscr{D}_{0}$ depending on the case, the proofs of those Propositions suffice to show that $[\Phi] \subseteq D \cdot \Phi$ and, assuming that $\mathscr{F}$ is smooth, that $[\Phi]_{\mathscr{F}}=D \cdot \Phi$. It then follows that $[\Phi]_{S}=[\Phi]$, since these get squeezed between $[\Phi]_{\mathscr{F}}$ and $D \cdot \Phi$.

And at the heuristic level, it seems safe to assume that $\mathscr{F}$ is smooth. For this equivalence relation arises as the transitive closure of the relation of being related by an element of a given set of flows on a manifold. And in the finite-dimensional setting, equivalence relations that arise in this way are always smooth [31, Theorem 4.1.a].

\section{Discussion}

Under the approach developed above gauge equivalence is identified with the weakest smooth equivalence relation on $\mathscr{S}$ stronger than the relation of being related by a spoiler. The approach was illustrated in Section 3 by being applied to an important special case: theories in which spatiotemporal symmetries constitute the only possible source of gauge equivalence. In this setting, it is possible to prove a number of results, which show that the present approach leads to intuitively plausible conclusions that match those of the standard Lagrangian approach. In particular, one finds the following. (i) For theories of the sort under consideration in which the spacetime metric is fixed, gauge equivalence is the trivial relation according to which every solution is equivalent only to itself. (ii) For well-behaved theories of this sort, gauge equivalent solutions are related by spatiotemporal symmetries. (iii) In well-behaved generally covariant theories with compact initial data surfaces solutions are gauge equivalent if and only if related by a spacetime diffeomorphism. (iv) In locally generally covariant theories featuring paradigmatically well-behaved asymptotic boundary conditions, one expects tp find that solutions are gauge equivalent if and only if related by a spacetime diffeomorphism asymptotic to the identity at infinity.

In this final section, two questions are addressed. (1) What are the prospects for extending the present approach to gauge equivalence? (2) What is the reason for the close relation between the notion of gauge equivalence developed here and the standard Lagrangian notion?

We have been working with a relatively narrow notion of a field theory, in which fixed and dynamical fields are assumed to be given by tensors on spacetime. This restriction was for convenience only: the definition of gauge equivalence given above in Section 2 makes sense in any theory in which fields and symmetries are suitably local on spacetime-e.g., in any theory in which field configurations are given by sections of (natural) bundles over spacetime. ${ }^{20}$ Given any such theory, analogues of Propositions 1 and 2 hold. In any theory in which fields are sections of (natural) vector bundles over spacetime, analogues of Propositions 3 and 4 can be proven using the techniques used above. ${ }^{21}$ But presumably analogues of these results hold far more generally.

20 For the notion of a natural bundle, see [22].

21 This is because the Lie derivative continue to be well-behaved in this setting [22, $\$ 6]$. 
One also expects that the approach should be well-behaved in application to non-spatiotemporal symmetries. Here the most important examples will be YangMills theories and their relatives - theories featuring among their fields a connection on a principal bundle over spacetime and with dynamics invariant under gauge transformations (vertical bundle automorphisms). At the formal level, one expects that after broadening slightly the notion of field theory as above and restricting attention to theories in which all spoilers are vertical bundle automorphisms, one should be able to adapt the arguments of Section 3.1 above to establish counterparts of the results proved there (with a connection $A$ playing the role of the metric $g$ and the group of gauge transformations playing the role of the group of spacetime diffeomorphisms).

Finally, it may be helpful to include a few remarks here about why the approach developed here agrees so closely with the Lagrangian approach in the cases discussed.

In the modern geometric version of the Lagrangian approach, one constructs as a by-product of the variational procedure an object $\omega$ that when fed a solution $\Phi \in$ $\mathscr{S}$ and two linearized solutions $\delta \Phi_{1}, \delta \Phi_{2}$ yields an $(n-1)$-form $\omega\left(\Phi, \delta \Phi_{1}, \delta \Phi_{2}\right)$ on $V$ (with $\omega$ linear in each $\delta \Phi_{i}$ ). Given a hypersurface $\Sigma \subset V$ one can consider $\Omega_{\Sigma}:=\int_{\Sigma} \omega$. This is a closed two-form on $\mathscr{S}$, so long as it is well-definedwhich it will be if $\Sigma$ is compact or there are well-behaved asymptotic boundary conditions in place and $\Sigma$ has good asymptotic behaviour.

One then finds that $\Omega_{\Sigma}$ depends only on the homology class of $\Sigma$ in $V$. So if we undertake to consider only $\Sigma$ that are adapted to the boundary conditions (if any) and that are homologous, then we can drop the subscript and view $\Omega$ as a closed two-form on $\mathscr{S}$. For example, we might decide to only allow $\Sigma$ that are Cauchy surfaces (with good asymptotic behaviour).

Given such a form, one can define a natural equivalence relation on $\mathscr{S}$ as follows. At each $\Phi \in \mathscr{S}$ we define the set of null vectors to be the subspace $\mathscr{N}_{\Phi} \subset T_{\Phi} \mathscr{S}$ consisting of those $\delta \Phi$ such that $\Omega(\Phi, \delta \Phi, \cdot)=0$. We call a curve in $\mathscr{S}$ null if all of its tangent vectors are null and we consider two solutions to be Lagrangian-gauge equivalent if they can be connected by a piecewise null curves. In well-behaved cases, the corresponding equivalence classes are submanifolds of $\mathscr{S} .^{22}$

Suppose that we have a field theory in which both the notion of gauge equivalence introduced above and the Lagrangian notion make sense and are wellbehaved. Let us denote the equivalence classes of the former by $[\Phi]$ and those of the latter by $\langle\Phi\rangle$. Since for any $\Phi \in \mathscr{S}$, both $[\Phi]$ and $\langle\Phi\rangle$ are submanifolds of $\mathscr{S}$, we can compare the two notions by comparing $T_{\Phi}[\Phi]$ and $T_{\Phi}\langle\Phi\rangle$ as subspaces of $T_{\Phi} \mathscr{S}$.

Let us call $\delta \Phi \in T_{\Phi} \mathscr{S}$ a spoiler vector at $\Phi$ if there exists a one-parameter group of spoilers $\left\{g_{t}\right\}$ such that $\delta \Phi=\left.\frac{d}{d t}\left(g_{t} \cdot \Phi\right)\right|_{t=0}$. Of course, relative to the definition of gauge equivalence given above, any spoiler vector $\delta \Phi$ at $\Phi$ is in $T_{\Phi}[\Phi]$. It is not hard to see that such a $\delta \Phi$ must be in $\langle\Phi\rangle$ as well. For there must be an initial data surface $\Sigma$ such that each $g_{t}$ acts as the identity on $\Sigma$. It follows that $\delta \Phi$ must vanish on $\Sigma$, so that $\Omega_{\Sigma}(\Phi, \delta \Phi, \cdot)=\int_{\Sigma} \omega(\Phi, \delta \Phi, \cdot)=0$, by the linearity of $\omega$ in $\delta \Phi$.

\footnotetext{
22 The set of null vectors fields is closed under Lie brackets (because $\Omega$ is closed) so one can invoke the Frobenius theorem so long as $\bigcup_{\Phi \in \mathscr{S}} \mathscr{N}_{\Phi}$ is a subbundle of $T \mathscr{S}$.
} 
In well-behaved cases, such as the ones studied above, one finds in fact that $T_{\Phi}[\Phi]$ is just the linear span of the spoiler vectors at $\Phi$. One hopes that something similar will hold in the Lagrangian case. In the cases of greatest interestgenerally covariant theories and theories invariant under gauge transformations of Yang-Mills type - the spoilers of a theory are contained in a group of symmetries that are localizable, in the sense that their infinitesimal generators are, roughly speaking, parameterized by arbitrary functions on $V .{ }^{23}$ One expects [27, p. 1281] that the null vectors at $\Phi$ point along the orbits of the subgroup of localizable symmetries with good asymptotic behaviour. ${ }^{24}$ And one further expects that this is equivalent to the claim that $\mathscr{N}_{\Phi}$ is the linear span of the spoiler vectors at $\Phi$-so that $[\Phi]=\langle\Phi\rangle$.

Acknowledgements I would like to thank Stefan Hollands, David Malament, Laura Ruetsche, Richard Thomas, and two anonymous referees for helpful conversations and advice. This work was supported by fellowships from the American Council of Learned Societies and from the Center for Advanced Study in the Behavioral Sciences

\section{References}

1. Andersson, L.: Momenta and reduction in general relativity. Journal of Geometry and Physics 4, 289-314 (1987)

2. Arms, J.: The structure of the solution set for the Yang-Mills equations. Mathematical Proceedings of the Cambridge Philosophical Society 90, 361-372 (1981)

3. Ashtekar, A., Bombelli, L., Reula, O.: The covariant phase space of asymptotically flat gravitational fields. In: M. Francaviglia (ed.) Mechanics, Analysis, and Geometry: 200 Years After Lagrange, pp. 417-450. Elsevier, Amsterdam (1991)

4. Ashtekar, A., Magnon, A.: Asymptotically anti-de Sitter space-times. Classical and Quantum Gravity 1, L39-L44 (1984)

5. Ashtekar, A., Romano, J.: Spatial infinity as a boundary of spacetime. Classical and Quantum Gravity 9, 1069-1100 (1992)

6. Chruściel, P.: Asymptotic symmetries of de Sitter spacetime. Acta Physica Polonica B 12, 719-737 (1981)

7. Chruściel, P., Isenberg, J.: Nonisometric vacuum extensions of vacuum maximal globally hyperbolic spacetimes. Physical Review D 48, 1616-1628 (1993)

8. Crnković, v., Witten, E.: Covariant description of canonical formalism in geometrical theories. In: S. Hawking, W. Israel (eds.) Three Hundred Years of Gravitation, pp. 676-684. Cambridge University Press, Cambridge (1987)

9. Deligne, P., Freed, D.: Classical field theory. In: P. Deligne, P. Etingof, D. Freed, L. Jeffrey, D. Kazhdan, J. Morgan, D. Morrison, E. Witten (eds.) Quantum Fields and Strings: A Course for Mathematicians, vol. 1, pp. 137-225. American Mathematical Society, Providence, RI (1999)

10. Dirac, P.A.M.: Lectures on Quantum Mechanics. Dover, New York (2001)

11. Frauendiener, J.: Conformal infinity. Living Reviews in Relativity 7(1) (2004). URL http://www.livingreviews.org/lrr-2004-1

12. Friedrich, H.: Einstein equations and conformal structure: Existence of anti-de Sitter spacetimes. Journal of Geometry and Physics 17, 125-84 (1995)

13. Friedrich, H.: Einstein's equation and conformal structure. In: L.J. Mason, K.P. Tod, S.T. Tsou, N.M.J. Woodhouse (eds.) The Geometric Universe: Science, Geometry, and the Work of Roger Penrose, pp. 81-98. Oxford University Press, Oxford (1998)

14. Friedrich, H.: Einstein's equation and geometric aysmptotics. In: N. Dadhich, J. Narlikar (eds.) Gravity and Relativity: At the Turn of the Millennium, pp. 153-176. Inter-University Centre for Astronomy and Astophysics, Pune (1998)

${ }^{23}$ See [9, Definition 2.93] and [24, p. 735] for precise versions of this notion.

${ }^{24}$ For the importance of the asymptotic condition, see [24]. 
15. Geroch, R.: Asymptotic stucture of space-time. In: F.P. Esposito, L. Witten (eds.) Asymptotic Stucture of Space-Time, pp. 1-105. Plenum Press, New York (1977)

16. Gotay, M., Nester, J., Hinds, G.: Presymplectic manifolds and the Dirac-Bergmann theory of constraints. Journal of Mathematical Physics 19, 2388-2399 (1978)

17. Hawking, S., Ellis, G.: The Large Scale Structure of Space-Time. Cambridge University Press, Cambridge (1973)

18. Henneaux, M., Teitelboim, C.: Asymptoticaly anti-de Sitter spaces. Communications in Mathematical Physics 98, 391-424 (1985)

19. Henneaux, M., Teitelboim, C.: Quantization of Gauge Systems. Princeton University Press, Princeton (1992)

20. Hollands, S., Ishibashi, A., Marolf, D.: Comparison between various notions of conserved charges in asymptotically AdS spacetimes. Classical and Quantum Gravity 22, 2881-2920 (2005)

21. Isenberg, J., Marsden, J.: A slice theorem for the space of solutions of Einstein's equations. Physics Reports 89, 179-222 (1982)

22. Kolár̆, I., Michor, P., Slovák, J.: Natural Operations in Differential Geometry. SpringerVerlag, Berlin (1993)

23. Lang, S.: Fundamentals of Differential Geometry. Springer-Verlag, Berlin (1999)

24. Lee, J., Wald, R.: Local symmetries and constraints. Journal of Mathematical Physics 31 , 725-743 (1990)

25. Olver, P.: Applications of Lie Groups to Differential Equations, 2nd edn. Springer-Verlag, Berlin (1993)

26. Pflaum, M.: Analytic and Geometric Study of Stratified Spaces. Springer-Verlag, Berlin (2001)

27. Reyes, E.: On covariant phase space and the variational bicomplex. International Journal of Theoretical Physics 43, 1267-1286 (2004)

28. Rosen, N.: Flat space and variational principle. In: B. Hoffmann (ed.) Perspectives in Geometry and Relativity: Essays in Honor of Václav Hlavatý, pp. 325-327. Indiana University Press, Bloomington (1966)

29. Sorkin, R.: An example relevant to the Kretschmann-Einstein debate. Modern Physics Letters A 17, 695-700 (2002)

30. Spradlin, M., Strominger, A., Volovich, A.: De Sitter space. In: C. Bachas, A. Bilal, M. Douglas, N. Nekrasov, F. David (eds.) Unity from Duality: Gravity, Gauge Theory and Strings, pp. 423-453. Springer-Verlag, Berlin (2003)

31. Sussmann, H.: Orbits of families of vector fields and integrability of distributions. Transactions of the American Mathematical Society 180, 171-188 (1973)

32. Torre, C., Anderson, I.: Symmetries of the Einstein equations. Physical Review Letters 70, 3525-3529 (1993)

33. Woodhouse, N.M.J.: Geometric Quantization, 2nd edn. Oxford University Press, Oxford (1991)

34. Zuckerman, G.: Action principles and global geometry. In: S.T. Yau (ed.) Mathematical Aspects of String Theory, pp. 259-284. World Scientific (1987) 